\title{
Heart Failure With Midrange Ejection Fraction: Prior Left Ventricular Ejection Fraction and Prognosis
}

\section{OPEN ACCESS}

Edited by:

Yasuhiro Ikeda,

Yamaguchi Prefectural Grand Medical

Center, Japan

Reviewed by:

Makoto Takei,

Keio University, Japan

Masashi Kanemoto,

Saiseikai Yamaguchi General

Hospital, Japan

Kenichi Hongo,

Jikei University School of

Medicine, Japan

*Correspondence:

Ying Liu

yingliu.med@gmail.com

Gary Tse

garytse86@gmail.com

tThese authors have contributed equally to this work and share first

authorship

Specialty section:

This article was submitted to Heart Failure and Transplantation,

a section of the journa

Frontiers in Cardiovascular Medicine

Received: 26 April 2021

Accepted: 29 June 2021

Published: 03 August 2021

Citation:

Zhang $X$, Sun Y, Zhang Y, Chen F, Zhang S, He H, Song S, Tse G and

Liu Y (2021) Heart Failure With Midrange Ejection Fraction: Prior Left Ventricular Ejection Fraction and Prognosis.

Front. Cardiovasc. Med. 8:697221.

doi: 10.3389/fcvm.2021.697221

\author{
Xinxin Zhang ${ }^{1 \dagger}$, Yuxi Sun ${ }^{1 \dagger}$, Yanli Zhang ${ }^{1}$, Feifei Chen ${ }^{1}$, Shuyuan Zhang ${ }^{1}$, Hongyan He ${ }^{1}$, \\ Shuang Song ${ }^{1}$, Gary Tse ${ }^{1,2 *}$ and Ying Liu ${ }^{1 *}$ \\ ${ }^{1}$ Heart Failure and Structural Cardiology Ward, The First Affiliated Hospital of Dalian Medical University, Dalian, China, ${ }^{2}$ Kent \\ and Medway Medical School, Canterbury, United Kingdom
}

Aims: Evidence-based guidelines for heart failure management depend mainly on current left ventricular ejection fraction (LVEF). However, fewer studies have examined the impact of prior LVEF. Patients may enter the heart failure with midrange ejection fraction (HFmrEF) category when heart failure with preserved ejection fraction (HFpEF) deteriorates or heart failure with reduced ejection fraction (HFrEF) improves. In this study, we examined the association between change in LVEF and adverse outcomes.

Methods: HFmrEF patients with at least two or more echocardiograms 3 months apart at the First Affiliated Hospital of Dalian Medical University between September 1, 2015 and November 30, 2019 were identified. According to the prior LVEF, the subjects were divided into improved group (prior LVEF < 40\%), stable group (prior LVEF between 40 and $50 \%$ ), and deteriorated group (prior LVEF $\geq 50 \%$ ). The primary outcomes were cardiovascular death, all-cause mortality, hospitalization for worsening heart failure, and composite event of all-cause mortality or all-cause hospitalization.

Results: A total of 1,168 HFmrEF patients (67.04\% male, mean age $63.60 \pm 12.18$ years) were included. The percentages of improved, stable, and deteriorated group were 310 (26.54\%), 334 (28.60\%), and 524 (44.86\%), respectively. After a period of follow-up, 208 patients (17.81\%) died and 500 patients met the composite endpoint. The rates of all-cause mortality were 35 (11.29\%), 55 (16.47\%), and $118(22.52 \%)$, and the composite outcome was 102 (32.90\%), 145 (43.41\%), and 253 (48.28\%) for the improved, stable, and deteriorated groups, respectively. Cox regression analysis showed that the deterioration group had higher risk of cardiovascular death $(\mathrm{HR}: 1.707,95 \% \mathrm{Cl}$ : 1.064-2.739, $P=0.027)$, all-cause death ( $\mathrm{HR} 1.948,95 \% \mathrm{Cl} 1.335-2.840, P=0.001)$, and composite outcome (HR 1.379, 95\% Cl 1.096-1.736, $P=0.006)$ compared to the improvement group. The association still remained significant after fully adjusted for both all-cause mortality $(\mathrm{HR}=1.899,95 \% \mathrm{Cl} 1.247-2.893, P=0.003)$ and composite outcome (HR: 1.324, 95\% Cl: 1.020-1.718, $P=0.035)$.

Conclusion: HFmrEF patients are heterogeneous with three different subsets identified, each with different outcomes. Strategies for managing HFmrEF should include previously measured LVEF to allow stratification based on direction changes in LVEF to better optimize treatment.

Keywords: heart failure, mid-range ejection fraction, prior, left ventricular ejection fraction, prognosis 


\section{INTRODUCTION}

Heart failure (HF) represents the final common pathway of different cardiac diseases and is a major cause of death among the elderly in many countries (1-4). Currently, risk management and treatment of HF mainly depend on current left ventricular ejection fraction (LVEF) in clinical practice $(5,6)$. In the latest European Society of Cardiology (ESC) guideline, HF was divided into $\mathrm{HF}$ with reduced ejection fraction (HFrEF), HF with mid-range ejection fraction ( $\mathrm{HFmrEF}$ ), and $\mathrm{HF}$ with preserved ejection fraction (HFpEF) based on LVEF (7). HFmrEF patients are encountered with an increasing frequency in contemporary HF clinics (8). The latest data show that the prevalence of HFmrEF in hospitalized patients ranged from 13 to $26 \%(9-11)$, while the prevalence in outpatients varied from 9 to $21 \%$ (1217). Nevertheless, previous studies mostly focused on HFrEF and HFpEF, with less attention paid to $\operatorname{HFmrEF}$ until now $(18,19)$. Consequently, less is known regarding the clinical characteristics of patients with HFmrEF, and with limited evidence on which to base recommendation for therapy (20).

Indeed, LVEF can be dynamic as the condition of the patient changes. To date, many investigators have been devoting to working on LVEF transition, exploring the incidence, predictors, and associations with outcomes of changes in LVEF in HF patients $(21,22)$. Some investigators have suggested that HFmrEF patients do not represent a distinct group, but rather represent a heterogeneous group of HFrEF and HFpEF patients, in whom a change in LVEF resulted in their being categorized as a unique subset of $\mathrm{HF}$ patients. In their view, HFmrEF represents a transitional state, and can easily progress to $\mathrm{HFpEF}$ or HFrEF. However, it must be pointed that transition into the HFmrEF category may also occur by either deterioration or improvement of LVEF. Up to now, there are few studies available describing their characteristics and clinical outcomes. In this study, we examined the association between changes in LVEF and adverse outcomes.

\section{MATERIALS AND METHODS}

\section{Study Population}

This retrospective cohort study was approved by the institutional review board of the First Affiliated Hospital of the Dalian Medical University. The inclusion criteria were patients admitted for acute decompensated $\mathrm{HF}$ at the First Affiliated Hospital of Dalian Medical University between September 1, 2015 and November 31, 2019. The exclusion criterion was a lack of prior echocardiography for comparison. Details of clinical characteristics, comorbidities, drug therapies, laboratory values, and echocardiography findings of the subjects were collected and recorded from Yidu Cloud. All procedures were conducted in accordance with the Declaration of Helsinki. As this was a retrospective research, no informed consents can be obtained.

\section{Classification of HF Cases}

We classified current HFmrEF patients as having (1) improved group (defined as any previously documented LVEF < 40\%), (2) stable group (defined as all previously documented LVEF between 40 and 50\%), and (3) deteriorated group (defined as at least one previously documented LVEF $\geq 50 \%$ ). The study flow chart was shown in Figure 1.

\section{Clinical Definitions}

$\mathrm{HF}$ is defined as a clinical syndrome with symptoms and/or signs caused by a structural and/or functional cardiac abnormality and corroborated by elevated natriuretic peptide levels and/or objective evidence of pulmonary or systemic congestion (23). According to echocardiographic data, patients with an EF from 40 to $50 \%$ were categorized as HFmrEF.

\section{Adverse Outcomes}

Cardiovascular death, all-cause death, and hospitalization for worsening $\mathrm{HF}$ were determined using the Yidu Cloud with complete follow-up through November 30, 2020. The composite endpoint was defined as all-cause hospitalization or all-cause mortality. If these data were unavailable, the status was ascertained by a telephone calling to the patients.

\section{Statistical Analysis}

Statistical analysis was performed using SPSS Statistical Software, Version 22.0 (SPSS Inc., Chicago, IL, USA). Patients' characteristics were summarized with continuous variables expressed as means \pm standard deviation and categorical variables presented as frequencies and percentages. Measurement data with a non-normal distribution were expressed as the median (interquartile range). The Kruskal-Wallis test was used for multi-group comparisons, and single-factor ANOVA was used for inter-group comparison. Characteristics were compared across HFmrEF groups using analysis of variance or chi-square tests, as appropriate. Kaplan-Meier analysis was used to describe the cumulative incidence of adverse events, and the long-rank test was used to compare differences.

Univariate and multivariate Cox proportional hazards regression models were used to investigate the risk factors of the endpoints. Covariates selected for multivariate Cox analysis come from either the one with a significance of $P<0.05$ in the univariate analysis or the one that had been proven to greatly affect the prognosis of HF (Supplementary Tables 1, 2), including age, male, coronary artery disease, hypertension, diabetes mellitus, cerebrovascular disease, ICD, beta-blockers, $\mathrm{ACEI} / \mathrm{ARB} / \mathrm{ARNI}$, spironolactone, loop diuretics, aspirin, statins, nitrates, hemoglobin, BNP, creatinine, plasma sodium, d-dimer, and time interval. The hazard ratios (HR) and 95\% confidence intervals (CI) compare clinical outcomes of cardiovascular death, all-cause death, hospitalization for worsening HF, and composite event of all-cause hospitalization or all-cause mortality for stable group compared with improved group (unadjusted and fully adjusted) and deteriorated group compared with improved group (unadjusted and fully adjusted). All $P$-values represent the significance of the HRs for stable group compared with improved group or deteriorated group compared with improved group. All values were two-tailed, and $P<0.05$ was considered statistically significant. 


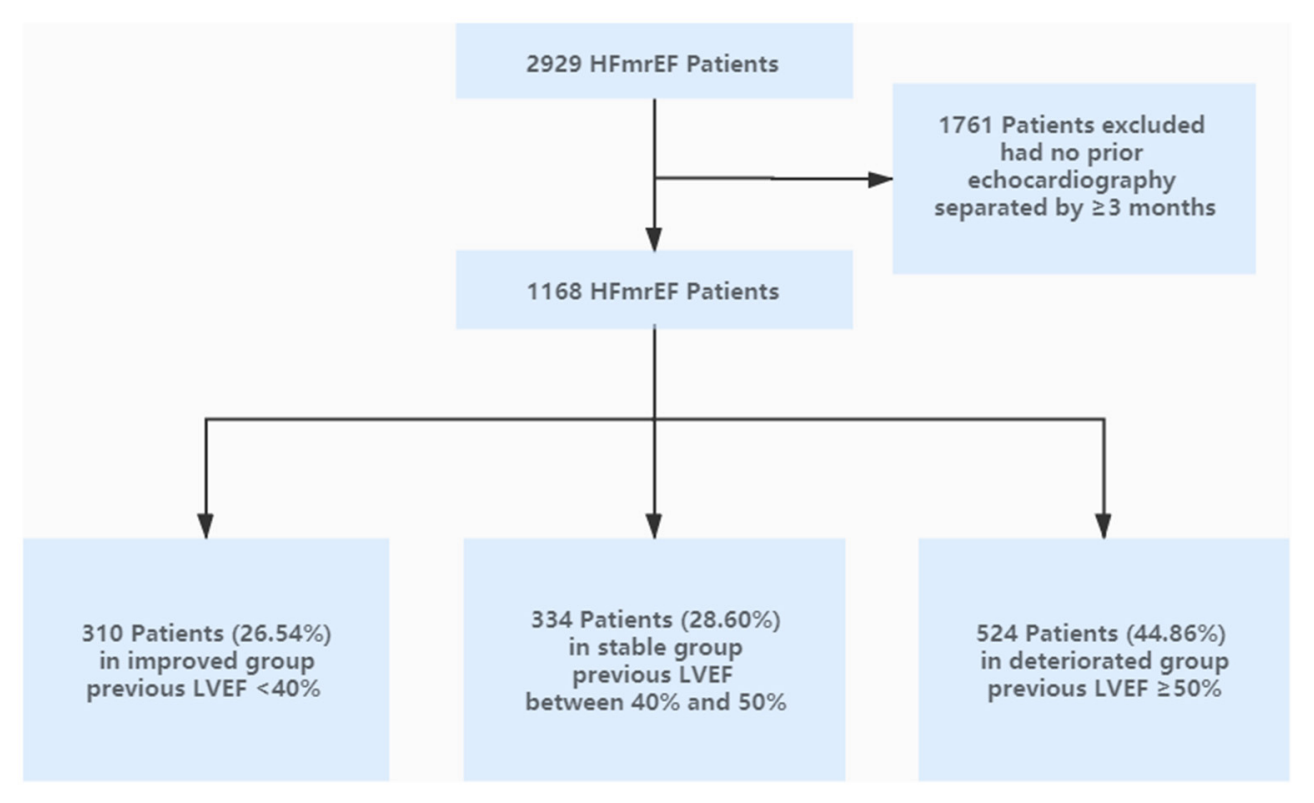

FIGURE 1 | Flow diagram of patient flow.

\section{RESULTS}

\section{Demographic and Clinical Characteristics}

Of 2,929 patients who had physician-diagnosed HFmrEF at our institution during September 1, 2015 and November 30, 2019, 1,761 patients were excluded due to the lack of availability of an echocardiogram separated by $>3$ months apart for comparison. A total of 1,168 patients were included (67.04\% male, mean age $63.60 \pm 12.18$ years). The percentages of improved, stable, and deteriorated group were $310(26.54 \%), 334(28.60 \%)$, and 524 (44.86\%), respectively. The flow chart indicating the inclusion and exclusion criteria was shown in Figure 1.

The baseline characteristics were shown in Table 1. In brief, patients in improved group were younger, had a higher proportion of males, and had a lower frequency of coronary artery disease, cancer, and hypertension compared with those in stable and deteriorated groups. There was no statistical difference in the proportion of NYHA class III-IV between the three groups at the prior echocardiogram. By contrast, improved group showed relative lower prevalence of NYHA class III-IV at the time of inclusion compared to the remaining two groups. Regarding medical therapies, patients in improved group were more likely to take angiotensin converting enzyme inhibitors (ACEI)/angiotensin receptor blockers (ARB)/angiotensin receptor neprilysin inhibitor (ARNI), betablockers, spironolactone, loop diuretics, and CRT compared to patients in the remaining two groups. As for laboratory data, the level of white blood cell, hemoglobin, platelet count, uric acid, and BNP in the improved group were significantly higher than other two groups. The average time interval between the two echocardiogram was 16 months. The interval in the deteriorated group was longer than that of the remaining two groups. Prior echocardiography findings showed that patients in improved group had higher left ventricular diameter and left atrial diameter, whereas with lowest value of interventricular septal thickness. Echocardiography findings at the time of inclusion indicated LVEF in all three subgroups fluctuated between 40 and 50 , and the value of LVEF in deteriorated group was higher than that of improved group. Moreover, improved group still had the highest left ventricular diameter among the three subgroups; nevertheless, there was no statistical significance across the three groups for the remainder of the parameters.

\section{Clinical Outcomes}

Over a median follow-up of 40.00 [25.00-53.00] months, there were 208 patients $(17.81 \%)$ deaths, and the percentages of improved, stable, and deteriorated group were 35 (11.29\%), 55 (16.47\%), and 118 (22.52\%), respectively. Five hundred patients met the composite endpoint (42.81\%), and the number were $102(32.90 \%), 145(43.41 \%)$, and $253(48.28 \%)$ for the improved, stable, and deteriorated groups, respectively. KaplanMeier analysis showed that the mortality and composite outcome in improved group was significantly lower than that in stable and deteriorated groups (Figures 2, 3). However, there was no statistical difference in the rates of cardiovascular death and hospitalization for worsening HF among the three subsets (Supplementary Figures 1, 2).

Cox regression analysis indicated that the deteriorated group showed a significantly higher risk of composite endpoint compared with patients in improved group (HR 1.379, 95\% CI $1.096-1.736, P=0.006)$. This difference was mainly due to trends toward increased risk of all-cause mortality (HR 1.948, 95\% CI 1.335-2.840, $P=0.001$ ). The association remained significant after adjustment for potential confounders for both mortality $(\mathrm{HR}=1.899,95 \% \mathrm{CI} 1.247-2.893, P=0.003)$ and 
TABLE 1 | Baseline demographics and clinical characteristics of the enrolled heart failure patients stratified by the directional change in LVEF.

\begin{tabular}{|c|c|c|c|c|c|}
\hline Characteristics & All patients $(n=1,168)$ & $\begin{array}{l}\text { Improved group } \\
\quad(n=310)\end{array}$ & $\begin{array}{l}\text { Stable group } \\
\quad(n=334)\end{array}$ & $\begin{array}{c}\text { Deteriorated } \\
\text { group }(n=524)\end{array}$ & $P$-value \\
\hline Age (years) & $63.60 \pm 12.18$ & $60.08 \pm 13.08^{\Psi \dagger}$ & $62.92 \pm 12.10^{\star \dagger}$ & $66.11 \pm 11.09^{\star \Psi}$ & $<0.0001$ \\
\hline Systolic blood pressure (mmHg) & $136.2 \pm 23.33$ & $133.0 \pm 22.22^{\dagger}$ & $136.3 \pm 23.25$ & $138.0 \pm 23.86^{\star}$ & 0.0118 \\
\hline Diastolic blood pressure $(\mathrm{mmHg})$ & $80.46 \pm 13.77$ & $81.85 \pm 14.11^{\dagger}$ & $80.77 \pm 13.23$ & $79.43 \pm 13.84^{*}$ & 0.0437 \\
\hline Heart rates & $82.21 \pm 22.02$ & $85.76 \pm 21.03^{\dagger}$ & $82.60 \pm 22.87$ & $79.85 \pm 21.79^{\star}$ & 0.0009 \\
\hline Body mass index $\left(\mathrm{kg} / \mathrm{m}^{2}\right)$ & $26.15 \pm 4.034$ & $26.66 \pm 4.1$ & $25.29 \pm 3.70$ & $26.27 \pm 4.15$ & 0.5814 \\
\hline Prior NYHA class III-IV (n, \%) & 322 (27.56\%) & $84(27.10 \%)$ & $95(28.44 \%)$ & $143(27.29 \%)$ & 0.9126 \\
\hline $\begin{array}{l}\text { NYHA class III-IV at the time of } \\
\text { inclusion ( } n, \%)\end{array}$ & 406 (34.76\%) & $89(28.70 \%)^{\Psi \dagger}$ & $130(38.92 \%)^{\star}$ & $187(35.68 \%)^{*}$ & 0.0204 \\
\hline \multicolumn{6}{|l|}{ Comorbidities } \\
\hline Diabetes mellitus $(n, \%)$ & 414 (35.45\%) & 97 (31.29\%) & $125(37.43 \%)$ & $192(36.54 \%)$ & 0.1980 \\
\hline Hypertension (n, \%) & 719 (61.56\%) & $166(53.55 \%)^{\Psi \dagger}$ & $211(63.17 \%)^{\star}$ & $342(65.27 \%)^{\star}$ & 0.0027 \\
\hline \multicolumn{6}{|l|}{ Therapy } \\
\hline ACEI/ARB/ARNI $(n, \%)$ & $652(55.82 \%)$ & $187(60.32 \%)^{\dagger}$ & $197(58.98 \%)^{\dagger}$ & $268(51.15 \%)^{* \Psi}$ & 0.0139 \\
\hline Aspirin (n, \%) & $683(58.48 \%)$ & 176 (56.77\%) & 209 (62.57\%) & 298 (56.87\%) & 0.1982 \\
\hline Beta-blockers $(n, \%)$ & 885 (75.77\%) & $266(85.81 \%)^{\Psi \dagger}$ & $267(79.94 \%)^{* \dagger}$ & $352(67.18 \%)^{* \Psi}$ & $<0.0001$ \\
\hline Digoxin $(n, \%)$ & $154(13.18 \%)$ & $62(20.00 \%)^{\dagger}$ & $36(10.78 \%)^{\star}$ & $56(10.69 \%)^{\star}$ & 0.0002 \\
\hline Loop diuretics $(n, \%)$ & 432 (36.99\%) & $145(46.77 \%)^{\Psi \dagger}$ & $121(36.23 \%)^{*}$ & $166(31.69 \%)^{\star}$ & $<0.0001$ \\
\hline Nitrates $(n, \%)$ & 438 (37.50\%) & $103(33.23 \%)^{\Psi}$ & $144(43.11 \%)^{*}$ & 191 (36.45\%) & 0.0280 \\
\hline Spironolactone ( $n, \%)$ & 596 (51.03\%) & $227(73.23 \%)^{\Psi \dagger}$ & $173(51.80 \%)^{\star \dagger}$ & $196(37.40 \%)^{\star \Psi}$ & $<0.0001$ \\
\hline Hemoglobin (g/L) & $136.9 \pm 21.64$ & $141.1 \pm 21.85^{\Psi \dagger}$ & $136.9 \pm 20.66^{\star}$ & $134.4 \pm 21.77^{\star}$ & $<0.0001$ \\
\hline Platelet (10^9/L) & $208.7 \pm 66.64$ & $222.4 \pm 80.98^{\Psi \dagger}$ & $202.0 \pm 59.32^{*}$ & $205.0 \pm 60.34^{*}$ & 0.0001 \\
\hline Creatinine $(\mu \mathrm{mol} / \mathrm{L})$ & $76.00(62.00,97.00)$ & $79(64.25,99.00)$ & $76.00(63.00,98.00)$ & $\begin{array}{l}74.00(61.00 \\
95.00)\end{array}$ & 0.6160 \\
\hline $\mathrm{UA}(\mu \mathrm{mol} / \mathrm{L})$ & $409.4 \pm 138.0$ & $440.9 \pm 161.1^{\Psi \dagger}$ & $412.5 \pm 131.9^{\star}$ & $390.3 \pm 124.5^{\star}$ & $<0.0001$ \\
\hline $\mathrm{Na}^{+}(\mathrm{mmol} / \mathrm{L})$ & $141.7 \pm 3.130$ & $141.6 \pm 3.169$ & $141.6 \pm 3.021$ & $141.7 \pm 3.179$ & 0.7728 \\
\hline Glu (mmol/L) & $6.351 \pm 2.614$ & $6.370 \pm 2.853$ & $6.373 \pm 2.489$ & $6.326 \pm 2.560$ & 0.9619 \\
\hline D-dimer ( $\mu \mathrm{g} / \mathrm{L})$ & $420(210.0,970.0)$ & $410(210.0,970.0)$ & $410(190.0880 .0)$ & $\begin{array}{c}455.0(230.0 \\
1,025)\end{array}$ & 0.2193 \\
\hline BNP level (ng/L) & $317.5(119.9,779.4)$ & $506.7(183.5,1,168)^{\dagger}$ & $337.4(127.0,922.1)^{\dagger}$ & $\begin{array}{c}231.2(90.40 \\
517.9)^{\star \Psi}\end{array}$ & $<0.0001$ \\
\hline \multicolumn{6}{|l|}{ Echocardiography parameters } \\
\hline Time interval (months) & $16.00(7.250,29.00)$ & $12.00(6.000,26.00)^{\dagger}$ & $13.50(7.000,27.00)^{\dagger}$ & $\begin{array}{l}19.00(10.00 \\
31.00)^{\star \Psi}\end{array}$ & $<0.0001$ \\
\hline \multicolumn{6}{|l|}{ Prior echocardiography findings } \\
\hline Left ventricular ejection fraction (\%) & $46.26 \pm 10.57$ & $32.41 \pm 5.626^{\Psi \dagger}$ & $43.61 \pm 2.711^{\star \dagger}$ & $56.16 \pm 3.088^{\star \Psi}$ & $<0.0001$ \\
\hline
\end{tabular}


TABLE 1 | Continued

\begin{tabular}{|c|c|c|c|c|c|}
\hline Characteristics & All patients $(n=1,168)$ & $\begin{array}{l}\text { Improved group } \\
\qquad(n=310)\end{array}$ & $\begin{array}{l}\text { Stable group } \\
\quad(n=334)\end{array}$ & $\begin{array}{c}\text { Deteriorated } \\
\text { group }(n=524)\end{array}$ & $P$-value \\
\hline Left ventricular diameter (mm) & $53.52 \pm 7.875$ & $59.69 \pm 7.213^{\Psi \dagger}$ & $54.22 \pm 6.706^{\star \dagger}$ & $49.61 \pm 6.466^{\star \Psi}$ & $<0.0001$ \\
\hline Interventricular septal thickness (mm) & $10.68 \pm 1.914$ & $10.38 \pm 1.661^{\Psi \dagger}$ & $10.80 \pm 1.951^{\star}$ & $10.78 \pm 2.007^{\star}$ & 0.0103 \\
\hline$E / e^{\prime}$ & $13.02 \pm 5.621$ & $13.46 \pm 5.383$ & $13.47 \pm 6.075$ & $12.38 \pm 5.372$ & 0.0534 \\
\hline Left ventricular diameter (mm) & $53.92 \pm 6.604$ & $56.24 \pm 6.259^{\Psi \dagger}$ & $54.78 \pm 6.759^{\star \dagger}$ & $51.84 \pm 6.076^{\star \Psi}$ & $<0.0001$ \\
\hline Left atrial diameter (mm) & $42.85 \pm 6.782$ & $42.28 \pm 6.630$ & $43.52 \pm 6.433$ & $42.77 \pm 7.079$ & 0.0682 \\
\hline Interventricular septal thickness (mm) & $10.76 \pm 1.998$ & $10.55 \pm 1.871$ & $10.73 \pm 2.153$ & $10.90 \pm 1.960$ & 0.0568 \\
\hline E/e' & $13.07 \pm 5.628$ & $12.19 \pm 5.645$ & $13.18 \pm 5.344$ & $13.49 \pm 5.770$ & 0.0668 \\
\hline
\end{tabular}

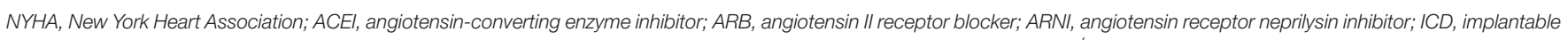

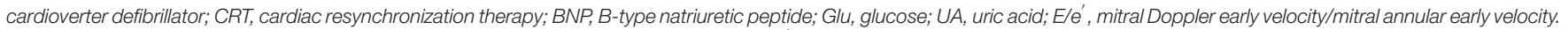
* is compared with Improved group $P<0.05, \Psi$ is compared with Stable group $P<0.05,{ }^{\dagger}$ is compared with Deteriorated group $P<0.05$.

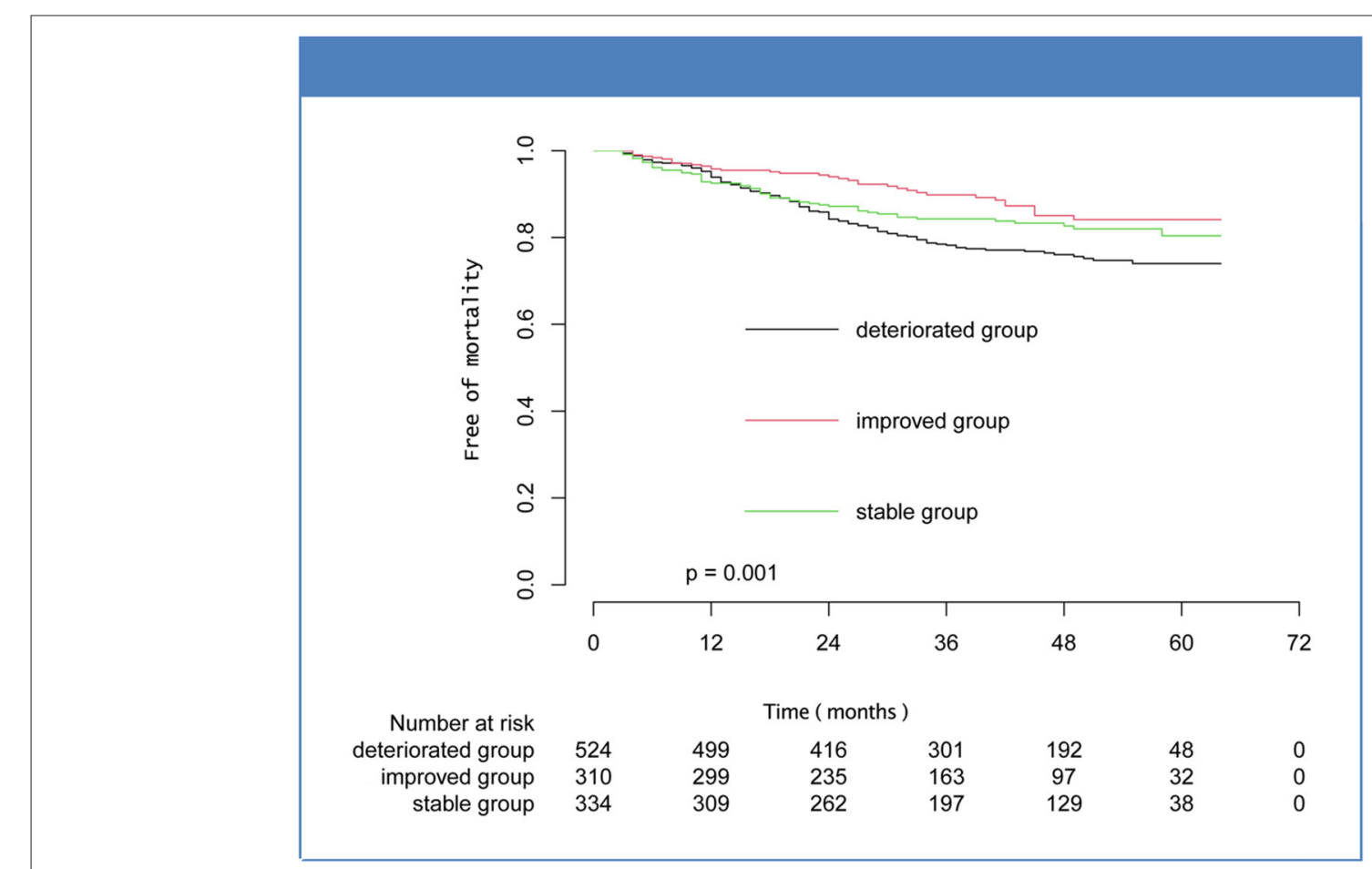

FIGURE 2 | Kaplan-Meier curves for mortality for the subsets of heart failure with midrange ejection fraction.

composite outcome (HR: 1.324, 95\% CI: $1.020-1.718, P=0.035)$. Moreover, compared to improved group, deteriorated group also experienced a 1.71-fold increase in risk of cardiovascular death (HR: $1.707,95 \%$ CI: $1.064-2.739, P=0.027$ ), albeit not reaching statistical significance in fully adjusted analysis. As with outcomes for hospitalization for worsening HF, HRs between the three subgroups did not show statistical differences. In addition, no significant differences in outcomes between patients in the improved and stable groups were seen for any of the endpoints in either unadjusted or fully adjusted analysis. The results were shown in Figure 4.

\section{DISCUSSION}

This study demonstrated that HFmrEF patients were a heterogeneous group of patients comprised of at least three different subsets. Additionally, the characteristics and clinical 


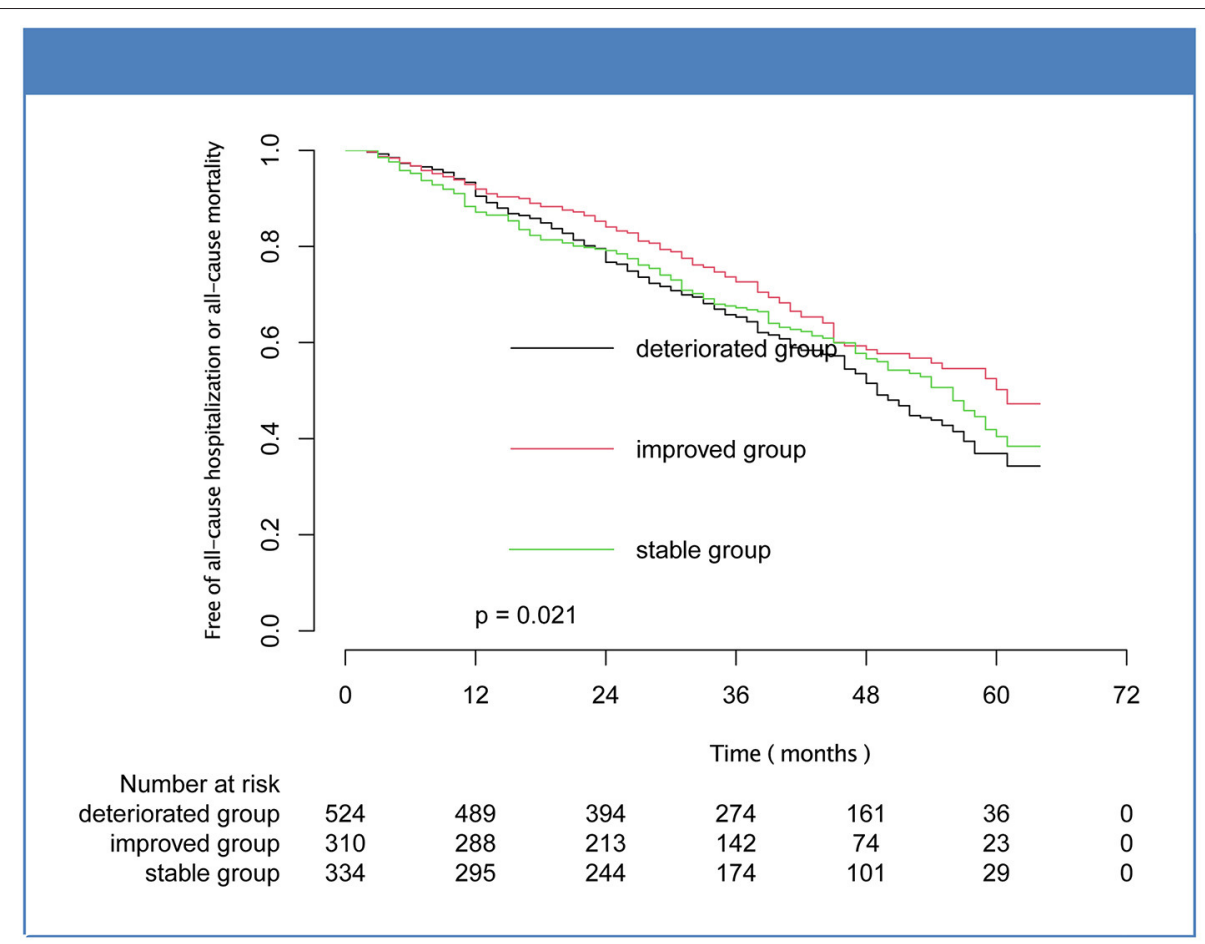

FIGURE 3 | Kaplan-Meier curves for composite outcome of mortality or hospitalization for the subsets of heart failure with midrange ejection fraction.

outcomes of HFmrEF patients among subgroups defined by the prior directional changes in LVEF are significantly different.

Risk stratification in $\mathrm{HF}$ is an important clinical problem $(24,25)$. Previous studies have elucidated that the demographics of patients with HFmrEF lied in between those of HFpEF and HFrEF patients, but in general were more similar to HFpEF patients, with a heavier burden of hypertension and atrial fibrillation/flutter $(10,12,13,26)$. Nevertheless, HFmrEF also resembled HFrEF showing a higher burden of ischemic heart disease (9, 27-29). In our study, we found that the HFmrEF cohort suffered from a heavy burden of comorbidities, such as hypertension $(61.56 \%)$, coronary heart disease $(54.20 \%)$, and atrial fibrillation/flutter (26.54\%). Our research also indicated that the characteristics of patients within HFmrEF subgroup were significantly different from the HFrEF and HFpEF subgroups. For example, patients in the deteriorated group were older, more female, and more likely to have hypertension, which were features consistent with HFpEF. By contrast, the deteriorated cohort had higher prevalence of coronary artery disease, which was in keeping with a HFrEF phenotype.

Regarding treatment, previous literatures suggested that HFmrEF patients received a mixture of medications indicated for both HFrEF and HFpEF patients (30, 31). Indeed, our study found that HFmrEF patients were prescribed medications recommended for HFrEF (digoxin, ACEI or ARB) as well as for HFpEF (calcium channel blockers). In our cohort, more than $50 \%$ patients received the traditional first-line agents of beta-blockers, ACEI/ARB, and aldosterone antagonist. Moreover, we found that patients in the improved group were more likely to take beta-blockers, ACEI/ARB/ARNI, spironolactone, and CRT than patients in the remaining two groups. The reason may be that neurohormonal blocking agents were only recommended for the patients with HFrEF but not HFpEF in $\mathrm{HF}$ management guidelines. Overall, these discrepancies underscored the considerable heterogeneity between patients in the HFmrEF population.

Notably, prior studies illustrated that the prognosis of HFmrEF patients was distinct from those of HFrEF and HFpEF. A 5-year follow-up of mortality showed that all-cause mortality in HFmrEF was higher than the rate of HFpEF patients, but lower than that of HFrEF patients $(32,33)$. However, HFmrEF mortality at 1 year after discharge was similar to that of $\operatorname{HFpEF}(10$, $34,35)$. The findings from four community-based longitudinal cohorts showed that age was an important clinical predictor of new onset HFmrEF (27). Meanwhile, a latest separate study demonstrated age $\geq 80$ years was associated with a higher risk of mortality within 1 year following discharge in the HFmrEF group compared with other HF types (35). In this study, our results also identified age as an independent risk factor for both mortality and composite outcome. Moreover, we found the adverse events of patients with HFmrEF varied considerably between subgroups and the clinical course was closely associated with the directional changes in LVEF that brought them into the mid-range. Unsurprisingly, patients in deteriorated group had a worse prognosis compared to other HFmrEF phenotypes, with a remarkably increased risk of a median follow-up of 40.00 months mortality and hospitalization, indicating the urgent need for careful follow-up of this group. The unfavorable outcomes may 


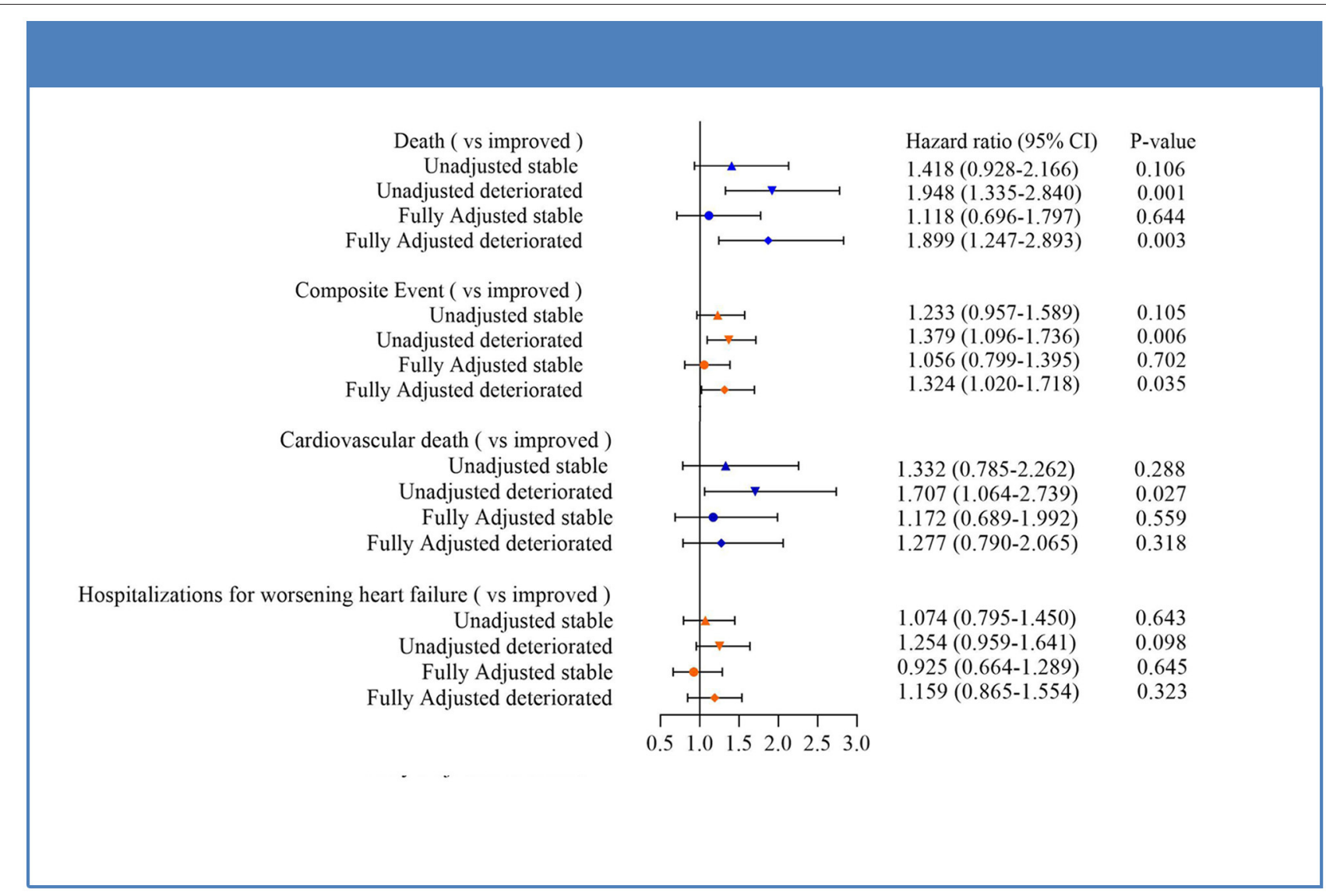

FIGURE 4 | Forest plot of clinical outcomes in HFmrEF subgroups.

be related to a large reduction of LVEF and a substantial increase in LV diameter in deteriorated group. The adverse alternations in cardiac structure and function are most likely due to the lower usage of guideline-directed medical therapy and the relatively high prevalence of coronary artery disease, as coronary artery disease was always associated with higher risk of mortality and worsening LVEF. In the large Improve Heart Failure Therapies in the Outpatient Setting registry, patients without prior myocardial infarction and non-ischemic HF etiology were both associated with a $>10 \%$ improvement in LVEF (36).

These findings suggested that for HFmrEF patients, previous changes in the direction of LVEF may provide important prognostic value, and clinicians should consider previous changes in LVEF when devising treatment plans.

\section{Limitations}

Nevertheless, we must note that this study still has several limitations. Firstly, considering the single-center nature of our study, the findings may not be generalizable to other settings. Secondly, the interval between the prior echocardiogram and the inclusion to the study was not exactly the same. Patients with echocardiography assessments within a short time period might have been less likely to exhibit a change in EF category. Although multivariate Cox regression models were applied to adjust for the interval between echocardiography assessments, residual confounding might have been a limitation. Thirdly, we can only obtain the medical record of patients hospitalized at our center, and we have no way of confirming when HF was first diagnosed, as this might have taken place at other hospitals. Thus, in this study, not every patient's echocardiogram time relative to initial HF diagnosis can be clearly recorded. Lastly, clinical outcomes were ascertained mainly depending on a telephone calling to the patients. Therefore, only a small number of patients in this cohort underwent the last follow-up echocardiography. In the near future, a large prospective cohort or a randomizedcontrolled study is necessary to understand the characteristics and evaluate the effects of drugs in HFmrEF population.

\section{Conclusions}

In conclusion, differences in the prevalence of risk factors and underlying etiology may generate different effects on LVEF transition, and thus different outcomes. The condition of HFpEF to HFmrEF is a dangerous and complex pathological process, which always implied worse clinical outcomes. These findings would remind clinicians to pay more attention to previous echocardiography results in HFmrEF patients, and to consider the impact of direction changes in LVEF on the prognosis of patients when planning management strategies. 


\section{DATA AVAILABILITY STATEMENT}

The raw data supporting the conclusions of this article will be made available by the authors, without undue reservation.

\section{ETHICS STATEMENT}

The studies involving human participants were reviewed and approved by the Institutional Review Board of Dalian Medical University. Written informed consent for participation was not required for this study in accordance with the national legislation and the institutional requirements.

\section{AUTHOR CONTRIBUTIONS}

$\mathrm{XZ}$ and YS were responsible for collecting clinical data and writing the paper. YZ and FC assisted YS in collecting data and conducting telephone follow-up. $\mathrm{HH}$ helped $\mathrm{YZ}$ with the follow-up. SS and SZ were responsible for the statistical analysis. YL and GT were responsible for revising the paper and

\section{REFERENCES}

1. Ito M, Wada H, Sakakura K, Ibe T, Ugata Y, Fujita H, et al. Clinical characteristics and mid-term outcomes of non-elderly obese patients with acute decompensated heart failure in Japan. Int Heart J. (2018) 59:766-71. doi: 10.1536/ihj.17-410

2. Yamaguchi T, Miyamoto T, Sekigawa M, Watanabe K, Hijikata S, Yamaguchi J, et al. Early transfer of patients with acute heart failure from a core hospital to collaborating hospitals and their prognoses. Int Heart J. (2018) 59:102633. doi: 10.1536/ihj.17-449

3. Yokokawa T, Ichijo Y, Houtsuki Y, Matsumoto Y, Oikawa M, Yoshihisa A, et al. Change of exhaled acetone concentration in a diabetic patient with acute decompensated heart failure. Int Heart J. (2017) 58:82830. doi: 10.1536/ihj.16-556

4. Yokokawa T, Sato T, Suzuki S, Oikawa M, Yoshihisa A, Kobayashi A, et al. Change of exhaled acetone concentration levels in patients with acute decompensated heart failure. Int Heart J. (2018) 59:80812. doi: 10.1536/ihj.17-482

5. Pathadka S, Yan VKC, Li X, Tse G, Wan EYF, Lau H, et al. Hospitalization and mortality in patients with heart failure treated with sacubitril/valsartan vs. enalapril: a real-world, population-based study. Front Cardiovasc Med. (2020) 7:602363. doi: 10.3389/fcvm.2020.602363

6. Tse G, Zhou J, Woo SWD, Ko CH, Lai RWC, Liu T, et al. Multi-modality machine learning approach for risk stratification in heart failure with left ventricular ejection fraction $</=45$. ESC Heart Fail. (2020) 7:3716-25. doi: 10.1002/ehf2.12929

7. Ponikowski P, Voors AA, Anker SD, Bueno H, Cleland JGF, Coats AJS, et al. 2016 ESC Guidelines for the diagnosis treatment of acute chronic heart failure: The Task Force for the diagnosis treatment of acute chronic heart failure of the European Society of Cardiology (ESC)Developed with the special contribution of the Heart Failure Association (HFA) of the ESC. Eur Heart J. (2016) 37:2129-200. doi: 10.1093/eurheartj/ehw128

8. Lakhani I, Leung KSK, Tse G, Lee APW. Novel mechanisms in heart failure with preserved, midrange, and reduced ejection fraction. Front Physiol. (2019) 10:874. doi: 10.3389/fphys.2019.00874

9. Kapoor JR, Kapoor R, Ju C, Heidenreich PA, Eapen ZJ, Hernandez $\mathrm{AF}$, et al. Precipitating clinical factors, heart failure characterization, and outcomes in patients hospitalized with heart failure with reduced, determining the research direction. All authors were involved in the drafting or revision of the manuscript.

\section{FUNDING}

This work was supported in part by National Science Foundation of China (No. U1908209).

\section{ACKNOWLEDGMENTS}

We would like to thank all the staff for their outstanding efforts in this work, especially those responsible for follow-up and statistics. The authors would like to thank Yidu Cloud (Beijing) Technology Co. Ltd. for their assistance in data searching, extraction, and processing.

\section{SUPPLEMENTARY MATERIAL}

The Supplementary Material for this article can be found online at: https://www.frontiersin.org/articles/10.3389/fcvm. 2021.697221/full\#supplementary-material borderline, and preserved ejection fraction. JACC Heart Fail. (2016) 4:46472. doi: 10.1016/j.jchf.2016.02.017

10. Cheng RK, Cox M, Neely ML, Heidenreich PA, Bhatt DL, Eapen ZJ, et al. Outcomes in patients with heart failure with preserved, borderline, and reduced ejection fraction in the Medicare population. Am Heart J. (2014) 168:721-30. doi: 10.1016/j.ahj.2014.07.008

11. Farmakis D, Simitsis P, Bistola V, Triposkiadis F, Ikonomidis I, Katsanos S, et al. Acute heart failure with mid-range left ventricular ejection fraction: clinical profile, in-hospital management, short-term outcome. Clin Res Cardiol. (2017) 106:359-68. doi: 10.1007/s00392-016-1063-0

12. Tsuji K, Sakata $Y$, Nochioka $K$, Miura M, Yamauchi T, Onose $T$, et al. Characterization of heart failure patients with mid-range left ventricular ejection fraction-a report from the CHART2 study. Eur J Heart Fail. (2017) 19:1258-69. doi: 10.1002/ ejhf. 807

13. Rickenbacher P, Kaufmann BA, Maeder MT, Bernheim A, Goetschalckx K, Pfister $\mathrm{O}$, et al. Heart failure with mid-range ejection fraction: a distinct clinical entity? Insights from the Trial of Intensified versus standard Medical therapy in Elderly patients with Congestive Heart Failure (TIME-CHF). Eur J Heart Fail. (2017) 19:1586-96. doi: 10.1002/ejhf.798

14. Vedin O, Lam CSP, Koh AS, Benson L, Teng THK, Tay WT, et al. Significance of ischemic heart disease in patients with heart failure and preserved, midrange, and reduced ejection fraction: a nationwide cohort study. Circ Heart Fail. (2017) 10:e003875. doi: 10.1161/CIRCHEARTFAILURE.117.003875

15. Pascual-Figal DA, Ferrero-Gregori A, Gomez-Otero I, Vazquez R, DelgadoJimenez J, Alvarez-Garcia J, et al. Mid-range left ventricular ejection fraction: clinical profile and cause of death in ambulatory patients with chronic heart failure. Int J Cardiol. (2017) 240:265-70. doi: 10.1016/j.ijcard.2017. 03.032

16. Ghio S, Guazzi M, Scardovi AB, Klersy C, Clemenza F, Carluccio E, et al. Different correlates but similar prognostic implications for right ventricular dysfunction in heart failure patients with reduced or preserved ejection fraction. Eur J Heart Fail. (2017) 19:873-9. doi: 10.1002/ejhf.664

17. Allen LA, Magid DJ, Gurwitz JH, Smith DH, Goldberg RJ, Saczynski J, et al. Risk factors for adverse outcomes by left ventricular ejection fraction in a contemporary heart failure population. Circ Heart Fail. (2013) 6:63546. doi: 10.1161/CIRCHEARTFAILURE.112.000180 
18. Sun Y, Wang N, Li X, Zhang Y, Yang J, Tse G, et al. Predictive value of H2 FPEF score in patients with heart failure with preserved ejection fraction. ESC Heart Fail. (2021) 8:1244-52. doi: 10.1002/ehf2.13187

19. Adamczak DM, Oduah MT, Kiebalo T, Nartowicz S, Beben M, Pochylski, et al. Heart failure with preserved ejection fraction-a concise review. Curr Cardiol Rep. (2020) 22:82. doi: 10.1007/s11886-020-01349-3

20. Wintrich J, Kindermann I, Ukena C, Selejan S, Werner C, Maack C, et al. Therapeutic approaches in heart failure with preserved ejection fraction: past, present, and future. Clin Res Cardiol. (2020) 109:107998. doi: 10.1007/s00392-020-01633-w

21. Gu J, Yin ZF, Zhang HL, Fan YQ, Zhang JF, Wang CQ. Characteristics and outcomes of transitions among heart failure categories: a prospective observational cohort study. ESC Heart Fail. (2020) 7:616-25. doi: 10.1002/ehf2.12619

22. Savarese G, Vedin O, D'Amario D, Uijl A, Dahlstrom U, Rosano $\mathrm{G}$, et al. Prevalence and prognostic implications of longitudinal ejection fraction change in heart failure. JACC Heart Fail. (2019) 7:306-17. doi: 10.1016/j.jchf.2018.11.019

23. Bozkurt B, Coats AJS, Tsutsui H, Abdelhamid CM, Adamopoulos S, Albert $\mathrm{N}$, et al. Universal definition and classification of heart failure: a report of the Heart Failure Society of America, Heart Failure Association of the European Society of Cardiology, Japanese Heart Failure Society and Writing Committee of the Universal Definition of Heart Failure: Endorsed by the Canadian Heart Failure Society, Heart Failure Association of India, Cardiac Society of Australia and New Zealand, and Chinese Heart Failure Association. Eur J Heart Fail. (2021) 23:352-80. doi: 10.1002/ejhf.2168

24. Lakhani I, Wong MV, Hung JKF, Gong M, Waleed KB, Xia Y, et al. Diagnostic and prognostic value of serum C-reactive protein in heart failure with preserved ejection fraction: a systematic review and meta-analysis. Heart Fail Rev. (2020). doi: 10.1007/s10741-020-09927-x. [Epub ahead of print].

25. Bazoukis G, Stavrakis S, Zhou J, Bollepalli SC, Tse G, Zhang Q, et al. Machine learning versus conventional clinical methods in guiding management of heart failure patients-a systematic review. Heart Fail Rev. (2021) 26:2334. doi: 10.1007/s10741-020-10007-3

26. Fonarow GC, Stough WG, Abraham WT, Albert NM, Gheorghiade M, Greenberg $\mathrm{BH}$, et al. Characteristics, treatments, and outcomes of patients with preserved systolic function hospitalized for heart failure: a report from the OPTIMIZE-HF Registry. J Am Coll Cardiol. (2007) 50:76877. doi: 10.1016/j.jacc.2007.04.064

27. Bhambhani V, Kizer JR, Lima JAC, van der Harst P, Bahrami H, Nayor M, et al. Predictors and outcomes of heart failure with mid-range ejection fraction. Eur J Heart Fail. (2018) 20:651-9. doi: 10.1002/ejhf.1091

28. Wang N, Hales S, Barin E, Tofler G. Characteristics and outcome for heart failure patients with mid-range ejection fraction. J Cardiovasc Med. (2018) 19:297-303. doi: 10.2459/JCM.0000000000000653

29. Savarese G, Orsini N, Hage C, Dahlstrom U, Vedin O, Rosano GMC, et al. Associations with and prognostic and discriminatory role of $n$ terminal pro-B-type natriuretic peptide in heart failure with preserved versus mid-range versus reduced ejection fraction. J Card Fail. (2018) 24:36574. doi: 10.1016/j.cardfail.2018.03.010

30. Bazoukis G, Thomopoulos C, Tse G, Tsioufis C. Is there a blood pressure lowering effect of MRAs in heart failure? An overview and meta-analysis. Heart Fail Rev. (2018) 23:547-53. doi: 10.1007/s10741-018-9689-9

31. Lopatin Y. Heart failure with mid-range ejection fraction and how to treat it. Card Fail Rev. (2018) 4:9-13. doi: 10.15420/cfr.2018:10:1

32. Hobbs FD, Roalfe AK, Davis RC, Davies MK, Hare R, Midlands Research Practices C. Prognosis of all-cause heart failure and borderline left ventricular systolic dysfunction: 5 year mortality follow-up of the Echocardiographic Heart of England Screening Study (ECHOES). Eur Heart J. (2007) 28:112834. doi: 10.1093/eurheartj/ehm102

33. Nadruz W Jr, West E, Santos M, Skali H, Groarke JD, Forman DE, et al. Heart failure and midrange ejection fraction: implications of recovered ejection fraction for exercise tolerance and outcomes. Circ Heart Fail. (2016) 9:e002826. doi: 10.1161/CIRCHEARTFAILURE.115.002826

34. Coles AH, Fisher K, Darling C, Yarzebski J, McManus DD, Gore JM, et al. Long-term survival for patients with acute decompensated heart failure according to ejection fraction findings. Am J Cardiol. (2014) 114:8628. doi: 10.1016/j.amjcard.2014.06.017

35. Coles AH, Tisminetzky M, Yarzebski J, Lessard D, Gore JM, Darling $\mathrm{CE}$, et al. Magnitude of and prognostic factors associated with 1-year mortality after hospital discharge for acute decompensated heart failure based on ejection fraction findings. J Am Heart Assoc. (2015) 4:e002303. doi: 10.1161/JAHA.115.002303

36. Wilcox JE, Fonarow GC, Yancy CW, Albert NM, Curtis AB, Heywood JT, et al. Factors associated with improvement in ejection fraction in clinical practice among patients with heart failure: findings from IMPROVE HF. Am Heart J. (2012) 163:49-56 e2. doi: 10.1016/j.ahj.2011.10.001

Conflict of Interest: The authors declare that the research was conducted in the absence of any commercial or financial relationships that could be construed as a potential conflict of interest.

Publisher's Note: All claims expressed in this article are solely those of the authors and do not necessarily represent those of their affiliated organizations, or those of the publisher, the editors and the reviewers. Any product that may be evaluated in this article, or claim that may be made by its manufacturer, is not guaranteed or endorsed by the publisher.

Copyright (c) 2021 Zhang, Sun, Zhang, Chen, Zhang, He, Song, Tse and Liu. This is an open-access article distributed under the terms of the Creative Commons Attribution License (CC BY). The use, distribution or reproduction in other forums is permitted, provided the original author(s) and the copyright owner(s) are credited and that the original publication in this journal is cited, in accordance with accepted academic practice. No use, distribution or reproduction is permitted which does not comply with these terms. 